J. Clin. Chem. Clin. Biochem.

Vol. 17, 1979, pp. 349-352

\title{
Bestimmung von $\alpha$-Amylase mit enzymatisch-kinetischer Methode auf dem ABA-100
}

\author{
Von W. Hohenwallner, E. Wimmer und R. Sommer
}

Laboratorium des Krankenhauses der barmherzigen Schwestern, Linz

(Eingegangen am 26. Juni/4. Dezember 1978)

Zusammenfassung: $\alpha$-Amylase wurde nach der enzymatischen Methode nach $H$. W. Schiwara ((1972) Z. Klin. Chem. Klin. Biochem. 10, 12-16) mit den Fertigreagentien der Fa. Smith Kline Instruments mit der Reaktionsfolge $\alpha$-Amylase- $\alpha$-Glucosidase-Hexokinase/Glucose-6-phosphatdehydrogenase am ABA-100 bestimmt.

Dabei wurden folgende Daten erhoben:

Der VK betrug in Kontrollsera und humanem Poolserum in der Serie 0,9-4,2\%, von Tag zu Tag 1,4-6,6\%.

Die Referenzwerte im Serum (212 Blutspender) betrugen 13-79 U/1 ( \pm 2 s), bei einem Mittel von 46 U/1. In Spontanharnen wurde keine Normalverteilung gefunden. Die Extremwerte bei Männern liegen bei 58-385 U/1, bei Frauen bei $7-318 \mathrm{U} / 1$.

Der Reaktionsablauf der kinetischen Methode wurde an Hand von Patienten- und Kontrollseren untersucht, wobei besonders auf den Einfluß der Glucose sowie auf die Linearität geachtet wurde.

Im Vergleich zur chromogenen Methode Amylochrom-Roche wurde bei Patientensera ein Korrelationskoeffizient von $r=0,975$, für Spontanharne ein $r=0,965$ gefunden.

\section{Determination of $\alpha$-amylase by an enzymatic kinetic method on the ABA-100.}

Summary: The enzymatic method of $H$. W. Schiwara (1972) Z. Klin. Chem. Klin. Biochem. 10, 12-16 (reagents by Smith Kline Instruments), using the enzymatic reaction sequence $\alpha$-amylase $-\alpha$-glucosidase - hexokinase/ glucose 6-phosphate dehydrogenase for the determination of $\alpha$-amylase was evaluated on the ABA-100.

The coefficient of variation for control sera and human pooled serum was $0.9-4.2 \%$ within series, and $1.4-6.6 \%$ day to day.

Reference values for a healthy population (212 blood donors) in sera were $13-79 \mathrm{U} / 1$ ( $\pm 2 \mathrm{SD}$ ), mean $46 \mathrm{U} / \mathrm{l}$. In catch urines the values did not show a normal distribution; the minimal and maximal range for men was 58-385 U/1, for women 7-318 U/1. The kinetic curve of the enzymatic test was measured and the influence of glucose and linearity studied.

In comparison with the enzymatic test, the chromogenic method Amlyochrom Roche was tested on the sera and urine of patients. The coefficient of correlation in sera was $r=0.975$, in urine $r=0.965$.

\section{Einfihrung}

Eine Reihe von enzymatisch-kinetischen Methoden zur Besstimmung der $\alpha$-Amylase sind in der Literatur beschrieben $(1-5)$. Von unserem Interesse war der Testkit (Fa. Smith Kline Instruments), der die $\alpha$-Amylase mit der enzymatischen Methode nach $H$. W. Schiwara (1) mißt. Wir untersuchtên Präzision und Richtigkeit des Tests und fuihrten eine Adaptation an den Analysenautomaten ABA-100 durch. Von Interesse waren weiterhin der Referenzbereich in Serum und Harn und schließlich vergleichende Untersuchungen mit der chromogenen Methode Amlyochrom-Roche.

\section{Material}

Für die Referenzwerterstellung im Serum wurden 103 männliche und 109 weibliche Probanden (Blutspender) im Alter von 18-40a mit der enzymatischen Methode untersucht. 
Da in der Routine Spontanharne zur Untersuchung kommen, wurden die Aktivitäten der $\alpha$-Amylase in im Rahmen von $\mathrm{Ge}$ sundenuntersuchungen gewonnenen Spontanharnen mit der chromogenen und enzymatischen Methode untersucht, und zwar 44 weibliche und 89 männliche Probanden im Alter von $15-45$ a.

Für den Methodenvergleich (enzymatischer Test und chromogene Methode) wurden 169 Sera und 134 Spontanharne von stationären Patienten beiderlei Geschlechts analysiert.

Qualitätskontrolle: für die chromogene Methode wurden die Kontrollsera Precinorm U (Ch. Nr. 710) Soll: 239-299-359 U/1, Validate A (Ch. Nr. 0149116) Soll: 432-540-648 U/1 und gepooltes Humanserum verwendet.

Für den enzymatischen Test: Eskalab (Ch. Nr. 5383) Soll: 24-33-44 U/1 und Eskalab (Ch. Nr. 4877) Soll: 443-503-563 U/1 und gepooltes Humanserum.

\section{Methodik}

Chromogene Methode (6)

Amylochrom-Roche

Enzymatische Methode (1)

a-Amylase (Smith Kline Instruments). Prinzip der Methode: Stärke nach Zulkowsky wird mit Hilfe der $\alpha$-Amylase der Probe zu Maltnse hydrolysiert, Maltose wird mit $\alpha$-Glucosidase zu Glucose gespalten, die Glucose mit Hexokinase/Glucose-6phosphatdehydrogenase bestimmt.

Geräteeinstellung am ABA-100:

Temperatur: $37^{\circ} \mathrm{C}$

Analysis time: $5 \mathrm{~min}$.

Filter: $380 / 340$

Mode Selection: RATE

Carousellrevolution: 3

Syringe Plate: 1:101

Direction: down

Probenvolumen: $5 \mu \mathrm{l}$

Kalibrationsfaktor: $\frac{5050}{\text { Filterfaktor }}$

Zero: $\frac{1800}{500 \times \text { Kalibrationsfaktor }}$

Nach der ersten Carousellrevolution wird das Gerät gestoppt und nach einer Inkubationszeit von $25 \mathrm{~min}$ werden zwei Messungen für die entsprechenden Absorptionsdifferenzen durchgeführt. Bei Glucosekonzentrationen von mehr als $250 \mathrm{mg} / 100 \mathrm{ml}$ sind die Proben mit $\mathrm{NaCl}$ (155 mmol/l) 1:5 zu verdünnen. Die Linearität wird bis $300 \mathrm{U} / \mathrm{l}$ angegeben.

\section{Enzymkinetik:}

Der Reaktionsablauf des enzymatischen Testes wurde am Eppendorf mit Kontronschreiber W/W 4301 bei $334 \mathrm{~nm}$ und $37^{\circ} \mathrm{C}$ aufgezeichnet, Schreibergeschwindigkeit $0,5 \mathrm{~cm} / \mathrm{min}$.

\section{Ergebnisse}

\section{Präzision und Richtigkeit}

Die Resultate in Serie und von Tág zu Tag sind aus Tabelle 1 ersichtlich.

\section{Referenżwerte im Serum}

Der \pm 2 s:Bereich betrug für den enzymatischen Test für ơ 12-78 U/1, für \% 15=79 U/1, für beide Kollektive $(\mathrm{N}=212) 13-79 \mathrm{U} / 1$ bei einem Mittel von $46 \mathrm{U} / \mathrm{l}$.

\section{Referenzwerte im Harn}

\section{Enzymatischer Test}

Es errechnet sich für die weiblichen Probanden $(\mathrm{N}=44)$ ein Bereịch von 4-318 und für die männlichen $(\mathrm{N}=89)$ $58-385 \mathrm{U} / 1$.

\section{Chromogene Methode}

Für die weiblichen Probanden (N. $=44$ ) wurde ein Bereich von $41-1350 \mathrm{U} / \mathrm{l}$ und für die männlichen $(\mathrm{N}=89)$ 42-1229 U/1 bestimmt.

\section{Methodenvergleich}

Die Berechnung der linearen Regression und Korrelation ist aus Tabelle 2 ersichtlich. Die Daten wurden gemäß des Resultates der chromogenen Methode im Serum $<500 \mathrm{U}$ und $>500 \mathrm{U} / 1$ unterteilt, im Spontanharn $<1500$ und $>1500 \mathrm{U} / \mathrm{l}$.

Es zeigt sich nach Korrektur der Extremwerte (außerhalb $\pm 2 \mathrm{~s}$ ) über den gesamten geprüften Bereich ein Korrelationskoeffizient von $\mathrm{r}=0,975$ im Serum und $\mathrm{r}=0,965 \mathrm{im}$ Harn .

Tab. 1. Prüfung der Präzision in Serie und von Tag zu Tag mit der chromogenen Methode Amylochrom-Roche und dem enzymatischen Test (SKI).

\begin{tabular}{|c|c|c|c|c|c|c|c|c|c|c|}
\hline \multirow[b]{2}{*}{ Methode } & \multirow[b]{2}{*}{ Probe } & \multicolumn{4}{|c|}{ Präzision in Serie } & & \multicolumn{3}{|c|}{ Präzision von Tag zu Tag } & \multirow[b]{2}{*}{ Technik } \\
\hline & & $\mathbf{N}$ & $\begin{array}{l}\bar{x} \\
(U / 1)\end{array}$ & (U/1) & $\begin{array}{l}\text { VK } \\
(\%)\end{array}$ & $\mathrm{N}$ & $\begin{array}{l}\bar{x} \\
(U / 1)\end{array}$ & $\stackrel{s}{(U / 1)}$ & $\begin{array}{l}\text { VK } \\
(\%)\end{array}$ & \\
\hline \multirow[t]{4}{*}{ Amylochrom } & Poolserum & 10 & 196,56 & 4,69 & 2,4 & 27 & 208,77 & 14,26 & 6,8 & \multirow{4}{*}{$\begin{array}{l}\text { Manuell } \\
\text { Kolorimetrie }\end{array}$} \\
\hline & $\begin{array}{l}\text { PNU 710 } \\
(239-299-359)\end{array}$ & - & - & - & - & 25 & 264,72 & 26,69 & 10,1 & \\
\hline & $\begin{array}{l}\text { Validate-A. } 149116 \\
(432-540-648)\end{array}$ & - & - & - & - & 52 & 482,48 & 44,77 & 9,3 & \\
\hline & 24 h-Harn & 10 & 192,33 & 4,33 & 2,25 & - & - & - & - & \\
\hline \multirow{4}{*}{$\begin{array}{l}\text { Enzymatischer } \\
\text { Test }\end{array}$} & Poolserum & 10 & 47,5 & 1,38 & 2,90 & 28 & 46,78 & 3,11 & 6,6 & \multirow{4}{*}{$\begin{array}{l}\text { ABA-1.00 } \\
\text { Optischer } \\
\text { Test }\end{array}$} \\
\hline & $\begin{array}{l}\text { Eskalab } 5083 \\
(24-33-42)\end{array}$ & 10 & 34,5 & 1,43 & 4,20 & 28 & 38,11 & 2,04 & 5,4 & \\
\hline & $\begin{array}{l}\text { Eskalab } 4877 \\
(443-503-563)\end{array}$ & 10 & 506,5 & 4,53 & 0,90 & 28 & 507,64 & 6,94 & 1,37 & \\
\hline & Spontanharn & 20 & $122 ; 55$ & 1,76 & 1,44 & - & - & - & - & \\
\hline
\end{tabular}


Tab. 2. Methodenvergleich, $x=$ chromogene Methode Amylochrom-Roche, $y=$ enzymatischer Test (SKI).

\begin{tabular}{|c|c|c|c|c|c|c|}
\hline & $\mathbf{N}$ & $\mathrm{U} / 1$ & $\overline{\mathbf{x}}$ & $\bar{y}$ & Regression & $\mathbf{r}$ \\
\hline Sera & $\begin{array}{r}153 \\
16\end{array}$ & $\begin{array}{l}<500 \\
>\quad 500\end{array}$ & $\begin{array}{r}189,58 \\
1.161,00\end{array}$ & $\begin{array}{r}42,08 \\
456,00\end{array}$ & $\begin{array}{l}y=0,26 x-7,0 \\
y=0,51 x-140\end{array}$ & $\begin{array}{l}0,868 \\
0,858\end{array}$ \\
\hline $\begin{array}{l}\text { nach Korrektur der } \\
\text { Werte außerhalb } \pm 2 \mathrm{~s}\end{array}$ & 161 & & 256,00 & 62,00 & $y=0,33 x-21$ & 0,975 \\
\hline Harne & $\begin{array}{r}106 \\
28\end{array}$ & $\begin{array}{l}<1.500 \\
>1.500\end{array}$ & $\begin{array}{r}764,00 \\
3.564,00\end{array}$ & $\begin{array}{l}225,00 \\
911,00\end{array}$ & $\begin{array}{l}y=0,28 x+10,2 \\
y=0,26 x-20,0\end{array}$ & $\begin{array}{l}0,926 \\
0,949\end{array}$ \\
\hline $\begin{array}{l}\text { nach Korrektur der } \\
\text { Werte außerhalb } \pm 2 \mathrm{~s}\end{array}$ & 130 & & $1.384,00$ & 373,00 & $y=0,15 x+20,0$ & 0,965 \\
\hline
\end{tabular}
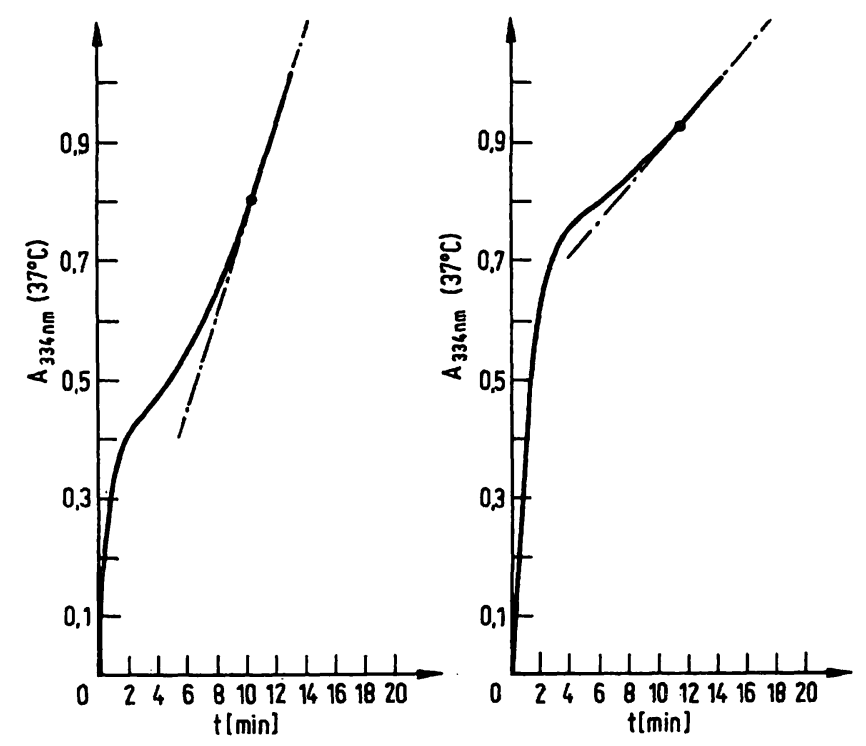

Abb. 1. Kinetik des enzymatischen Testes, gemessen a) im Patientenserum (956 U/l, unverdünnt).

b) im Kontrollserum Eskalab 4877 , SKI abnormal (443-503-560 U/1) bei $37^{\circ} \mathrm{C}$ und $334 \mathrm{~nm}$, Schreibergeschwindigkeit $0,5 \mathrm{~cm} / \mathrm{min}$.

\section{Enzymkinetik}

Die am Photometer Eppendorf untersuchten Aktivitäten im normalen und abnormalen Kontrollserum bzw. im Patientenserum zeigen das Verhalten der primären Umsetzung der präformierten Glucose und anschließend die hydrolytische Amylasewirkung und den damit verbundenen Glucoseumsatz (Abb. 1). Im normalen Kontrollserum beginnt nach etwa 15 min Inkubation die Linearität, während im abnormalen Kontrollserum, das wegen des hohen Glucosegehaltes $1: 2$ verdünnt wurde, die Linearität sicher nach 20 min einsetzt. In Abbildung 1 wurde dasselbe Kontrollserum unverdünnt aufgezeichnet, hier wird die Messung durch den hohen Umsatz an NADH am Beginn der Reaktion gestört. Grund- sätzlich wird von der Herstellerfirma eine Inkubationszeit von 25 min angegeben, um sicher im linearen Bereich zu messen.

\section{Diskussion}

Versucht man eine zusammenfassende Kritik bezüglich dieses enzymatischen Testes zu üben, so zeigen unsere Erfahrungen eine durchaus praktikable Methode, die sich sehr leicht an den ABA-100 adaptieren läßt, womit der Wunsch einer Automatisation der Amylasebestimmung erfüllt ist. Die Methode zeigt damit ein hohes $\mathrm{Maß}$ an Präzision, gemessen in Kontrollsera und menschlichem Poolserum und Harn. Im Vergleich zur chromogenen Methode sind einige Nachteile beseitigt, wie das Zentrifugieren, Messen und das Problem der Standardisierung. Eine Angabe in internationalen $U / 1$ ist möglich. Voraussetzung für die manuelle Durchführung des Testes ist ein Präzisionsphotometer mit genauer Temperierung, im Normbereich werden nur geringe Absorptionsdifferenzen gemessen.

Ein Nachteil des Testes ist die relativ lange Inkubationszeit von $25 \mathrm{~min}$, um sicher im linearen Bereich zu messen. Für die Notfalluntersuchung scheint uns der zeitliche Aufwand zu groß. Bezüglich der Vergleichbarkeit der Methode $z$ wischen verschiedenen Laboratorien liegen bisher noch keine Resultate vor, hingegen haben wir bei der chromogenen Methode, was unsere Erfahrung betrifft, durchweg gute Ergebnisse erzielt.

Der Weg, die Amylase mit Hilfe des enzymatisch-kinetischen Testes zu messen, ist sicherlich als Fortschritt zu bezeichnen, wobei, wie die Methode nach Pierre et al $(3,5)$ mit kurzer lag-Phase und hoher Linearität aufzeigt, der vorliegende Test (Smith Kline Instruments) nach Änderung des Substrates (z. B. Oligosaccharide wie Maltopentaose ect.) und der chemischen Reaktion verbesserungsfähig wäre. 


\section{Literatur}

1. Schiwara, H. W. (1972), Z. Klin. Chem. Klin. Biochem. 10, $12-16$.

2. Tietz, N. W., Whang, S. J. \& Miranda, E. (1972), Abstract presented at Int. Seminar and Workshop in Enzymology, Chicago, May 21-24, p. 2-9 bis 2-12.

3. Pierre, K. J., Tung, K. K. \& Nadj, H. (1976), Clin. Chem. 23, 1219.

4. Guilbault, G. G. \& Rietz, E. B. (1976), Clin. Chem. 22, 1702-1704.

5. Hanson, N. Q. \& Yasmineh, W. G. (1978), Clin. Chem. 24, 762-768.

6. Dalal, F. R. \& Winsten, S. (1971), Clin. Chim. Acta 32, 327-332.

Prim. Dr. W. Hohenwallner Langgasse 16

A-4010 Linz 